\title{
Inter-granular residual stresses in polycrystalline aggregates: finite element modelling and diffraction post-processing
}

\author{
Xu Song ${ }^{1}$, Shu Yan Zhang ${ }^{1}$, Daniele Dini ${ }^{2}$, Alexander M. Korsunsky ${ }^{1, a}$ \\ ${ }^{1}$ Dept of Engineering Science, University of Oxford, Parks Road, Oxford OX1 3PJ, England \\ ${ }^{2}$ Dept of Mechanical Engineering, Imperial College, Exhibition Road, London SW7 2AZ, England \\ aalexander.korsunsky@eng.ox.ac.uk
}

Keywords: residual stress, X-ray diffraction, diffractometer, polycrystal, elasticity, plasticity

\begin{abstract}
Most models based on continuum mechanics do not account for inhomogeneities at the micro-scale. This can be achieved by considering a representative volume of material and using (poly)crystal elasto-plastic deformation theory to model the effects of grain morphology and crystallographic orientation. In this way, the relationship between the macroscopic stress state and the stress state at the grain level can be investigated in detail. In addition, this approach enables the determination of the inhomogeneous fields of plastic strain, the identification of regions of localised plasticity (persistent slip bands), grain level shakedown, and the prediction of fatigue crack initiation using energy dissipation at the micro-scale. Elastic anisotropy is known to promote earlier onset of yielding, and to increase the magnitude of intergranular residual stresses. The effect of hardening behaviour of different slip systems on intergranular residual stresses is more subtle, as discussed in the text. The present study focuses on the analysis average intergranular residual strains and stresses that arise within the polycrystal aggregate following the application of single or cyclic external loading. These residual strains can also be evaluated experimentally using diffraction of penetrating radiation, e.g. neutrons or high energy X-rays, allowing comparisons with the model predictions to be made.
\end{abstract}

\section{Introduction}

Residual stresses are often classified into macroscopic and microscopic, depending on the scale of consideration. The question of scale is so intricately linked to the averaging process employed that often they are used almost interchangeably. However, e.g. in multi-phase materials it is possible to distinguish stresses in different phases, so that if selective averaging is performed even over macroscopic volumes, different results emerge depending on the phase considered.

Diffraction as a technique for the analysis of crystal structure and strain possesses several distinct advantages. It combines flexibility, accuracy and availability with the non-destructive nature of the measurement process, making it particularly attractive in cases when the sample is required for subsequent loading, or other testing and analysis. Diffraction measurements are naturally selective, not only in terms of crystallographic phase, but also in terms of crystal orientation. In many applications of diffraction for crystal structure analysis sampling volumes are nearly macroscopic, and therefore contain many grains (crystallites). Interpreted data regarding crystal structure - e.g., interplanar lattice spacings - therefore provide the kind of mesoscopic average values that one could otherwise expect to be obtained from phase-selective averaging.

It is therefore logical to seek and develop theoretical models of polycrystal deformation that would be capable of providing outputs from numerical simulations that are directly comparable with the results of diffraction measurements. Such models require to be informed about the microstructure, in terms of the statistics of grain orientation (texture), grain size distribution, modes of elastic and plastic deformation, etc.

The finite element formulation used in the present study uses the crystal plasticity FE framework due to Manonukul and Dunne [1], that is based on the formulations of Hill and Rice [2], and Asaro [3], with modifications and extensions [4] introduced to account for grain-level anisotropy of elastic properties, and to allow fully three-dimensional consideration of FCC, BCC and HCP crystal structures, as well as plane strain and plane stress approximations. The developments also 
incorporate the capability of diffraction post-processing, enabling direct comparison with experimental measurements of orientation-specific mesoscopic intergranular strains using neutron or high energy X-ray diffraction.

\section{Experimental measurement of mesoscopic average residual elastic strains}

Time-of-flight (TOF) neutron diffraction and energy-dispersive high energy X-ray diffraction allow simultaneous collection of multiple diffraction peaks related to mesoscopic ensembles of grains sharing close orientation with respect to the scattering vector. The patterns collected by the timeresolving pr energy-dispersive detectors can be interpreted either by crystal structure refinement of the entire multi-peak pattern (e.g. [5]), or by single peak fitting in order to extract individual mesoscopic grain orientation-specific strain averages (e.g. [6]).

In the present study we consider the data from neutron diffraction measurements obtained during reversed (tensile-compressive) cyclic deformation of FCC nickel-base superalloy C263 on the ENGIN-X time-of-flight spectrometer at ISIS, Rutherford Appleton Laboratory, Chilton, Oxon, UK. The samples, in the form of threaded cylindrical dog-bones, were loaded in situ using the setup illustrated in Fig. 1(a). A clip gauge mounted on the sample allowed the tests to be carried out under strain control. The loading frame is placed at $45^{\circ}$ to the incident beam, while the two detectors (marked " $L "$ and " $R$ " in the Figure) are mounted at the scattering angles of approximately $90^{\circ}$. The two detectors therefore correspond to the scattering vectors coinciding with the longitudinal (parallel to loading) and transverse sample directions. Data were collected at a sufficient number of points within each cycle so as to obtain the detailed response of orientation-specific grain groups.

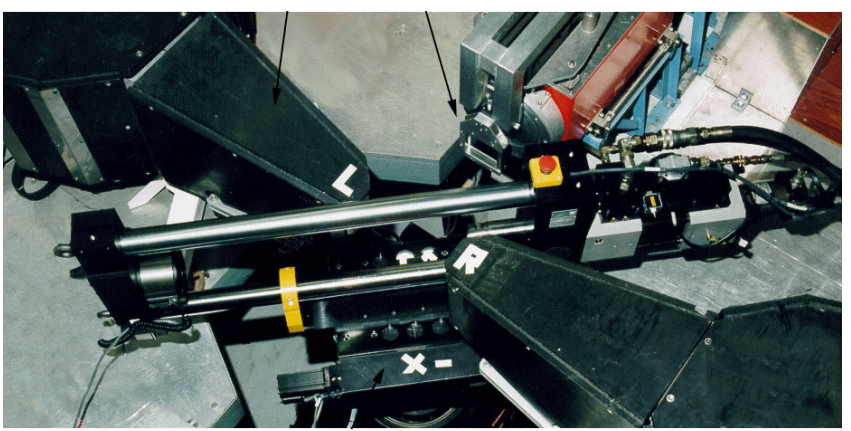

Fig. 1(a). In situ setup for fully reversed (tensioncompression) cyclic loading on the ENGIN-X spectrometer at ISIS (UK), showing the horizontally mounted $50 \mathrm{kN}$ servohydraulic Instron loading rig.

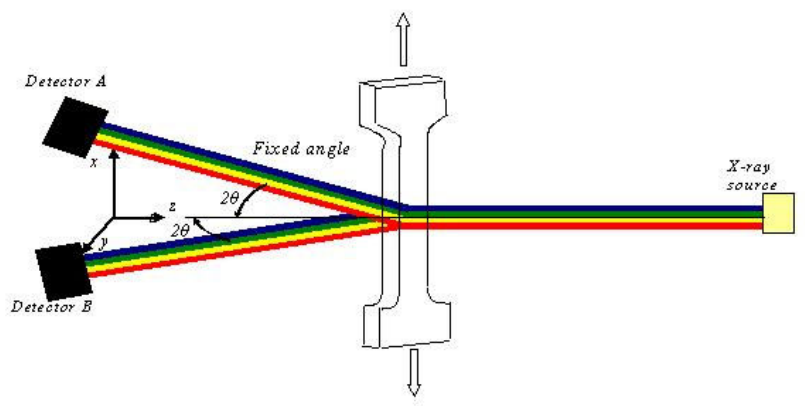

Fig. 1(b). Schematic illustration of the setup employed on the high energy synchrotron instrument ID15A at ESRF, Grenoble. The two detectors are offset by a small angle $2 \theta$ with respect to the incident beam in the horizontal and vertical scattering planes, respectively.

A similar setup was employed at ID15A high energy X-ray instrument at the ESRF (Grenoble, France), illustrated schematically in Fig. 1(b). Since the scattering angle becomes small when high energy X-rays are used, it was assumed that detector A and B corresponded to the scattering vectors lying in the plane of the sample, and being vertical and horizontal, respectively. The samples used in this case were made from Ti-6Al-4V aerospace titanium alloy. They loading schedule was in this case purely tensile, but incorporated repeated unloads, allowing measurements of residual elastic strains to be made.

Mesoscopic average lattice spacing within a grain group with the $h k l$ plane normal oriented in the direction of the scattering vector was computed using Bragg's law:

$$
2 d_{h k l} \sin \theta=\lambda \text {. }
$$

\section{Longitudinal residual strains in cyclically deformed C263 samples}

The macroscopic stress-strain curve for the sample investigated is illustrated in Fig. 2(a). The markers indicate the points at which diffraction data were collected in the first loading cycle. The results are presented for the longitudinal $h k l$ strains collected during in situ loading through three initial cycles of deformation in Fig. 2(b). Note that macroscopic applied stress is plotted against the vertical axis, and that the magnitude of error of strain evaluation is comparable with the size of the markers. The results indicate significant difference in the elastic response of different grain 
orientations, as evidenced by the different slope of macrostress vs microstrain curves. The difference in the plastic response is also apparent: while 111 and 311 loading plots represented in this way appear closed, both 200 and 220 loops show significant non-linearity.

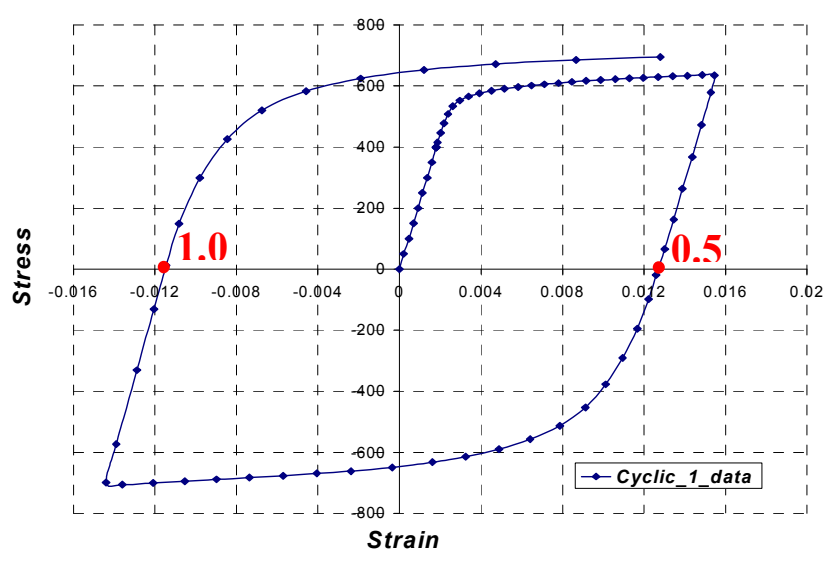

Fig. 2(a). Macroscopic stress-strain curve for Ni-base superalloy C263 during in situ cycling at ENGIN-X. Markers show when diffraction patterns were collected. Cycle numbers for unloading analysis also are shown.

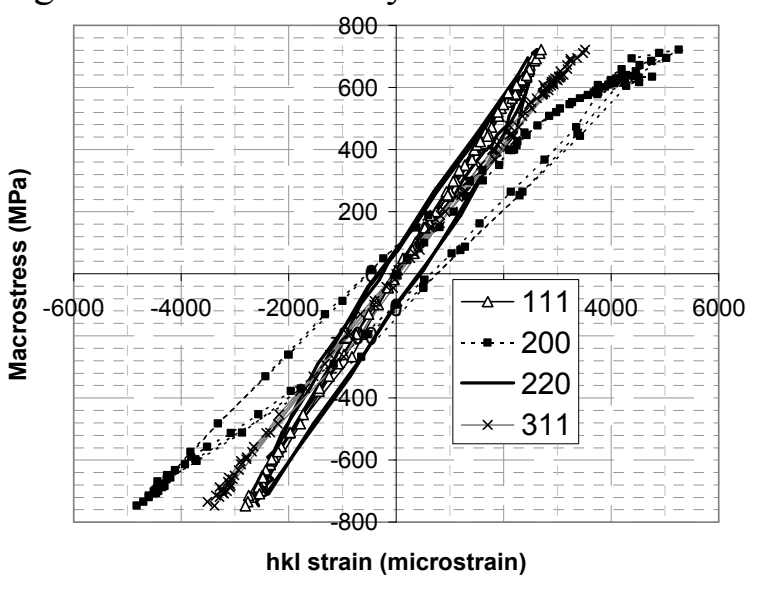

Fig. 2(b). Macrostress vs microstrain hysteresis loops for $h k l$ grain groups 111, 200, 220 and 311, showing significant differences in both elastic (slope) and plastic (loop width) response to applied loading.

The interpretation of these differences in response as a function of grain orientation is discussed elsewhere (e.g. [4]). Here we are particularly interested in the orientation specific residual elastic strains, that can be readily extracted from the data presented in Fig. 2(b). Indeed, even though in situ loading ws conducted under macroscopic strain control, the values of microstrains corresponding to zero macroscopic stress can be readily extracted by interpolation, noting that the macrostressmicrostrain relationship is very close to being linear in this range (see Fig. 2b).

Fig. 3 presents the evolution history of $h k l$ residual elastic strains as a function of the number of cycle considered ( 0 corresponds to the initial state, 0.5 corresponds to the first unload, i.e. first halfcycle, etc.) Several trends are immediately apparent. The two first peaks, 111 and 200, correspond to extremes of response: while 111-oriented grains go into compression after first unloading, 200oriented grains experience residual tension, as well as 311-oriented grains, while 220-oriented grains acquire almost no residual strain. During subsequent cycles of loading and unloading the 111 and 200-oriented grains remain opposite in sign and show widest variation of residual strains between tension and compression, while other orientations experience less extreme variations. These observations are in agreement with previously reported results and views about the significance of the so-called difference strain, and its close relationship with plastic strain history in polycrystalline samples [7]. Similar evolution behaviour arises from the analysis of FE crystal plasticity models, confirming that the grain level processes occurring within polycrystalline aggregates are captured correctly. If two grains of orientations close to 111 and 200 find themselves in a neighbouring position, e.g. sharing a grain boundary, then it is reasonable to deduce that this considerable relative straining may be particularly damaging in terms of promoting crack initiation. In fact, such grain junctions may in fact control initiation fatigue life in polycrystalline samples. 


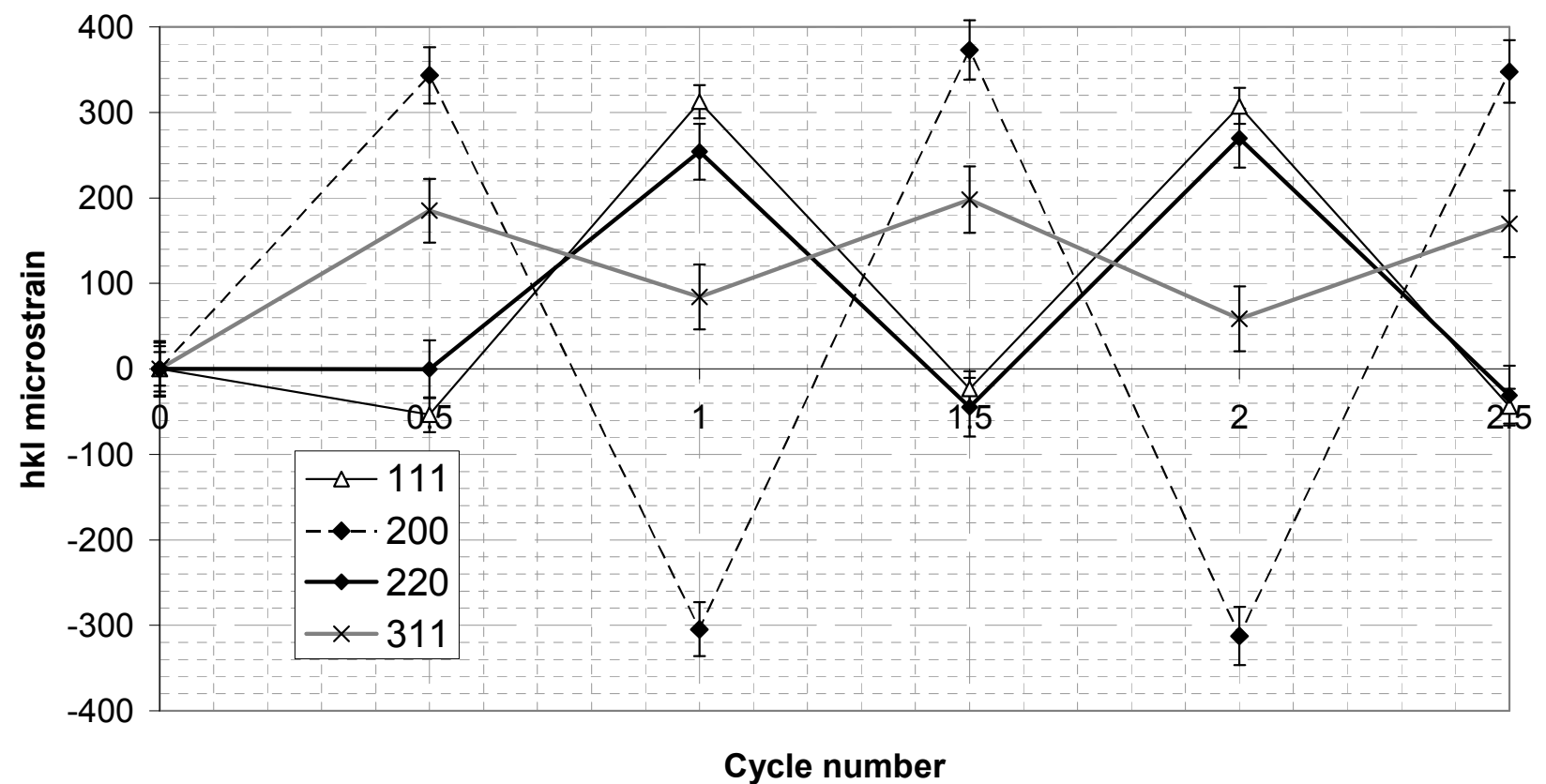

Fig. 3. The evolution of orientation-specific residual elastic strains as a function of the number of cycles.

\section{Longitudinal and transverse residual stresses in Ti-6Al-4V tensile samples}

Tensile test specimens made from the Ti-6Al-4V alloy in the form of dog-bones with the cross section of $3 \times 3 \mathrm{~mm}^{2}$ were mounted in the Instron universal testing machine on the high energy X-ray scattering beam line (ID15) at European Synchrotron Radiation Facility (ESRF) in Grenoble, France. The in-situ loading experiments were performed in the white beam energy dispersive mode, allowing multiple Bragg peaks to be measured simultaneously. The gauge volume was defined using $0.1 \mathrm{~mm}$ slits in both the incident and diffracted beam paths, and $2 \theta$ scattering angle of $5^{\circ}$. The sampling volume in the sample was a parallelepiped of $0.1 \times 0.1 \mathrm{~mm}^{2}$ cross section and $2.3 \mathrm{~mm}$ long. The sample was loaded incrementally in $500 \mathrm{~N}$ steps and held at the prescribed load level while diffraction data were collected. The data acquisition time was 120 seconds. The loading axis was normal to the incident beam. Twin-detector setup allowed simultaneous evaluation of strains in the loading direction, and the other direction normal to the beam and the loading direction.

Deformation was simulated using the polycrystal elastic-plastic FE model, and the results analysed using diffraction post-processing routines [4]. Good agreement was found between the trends in model predictions (continuous lines) and experiment data (markers) shown in Fig. 4(a).

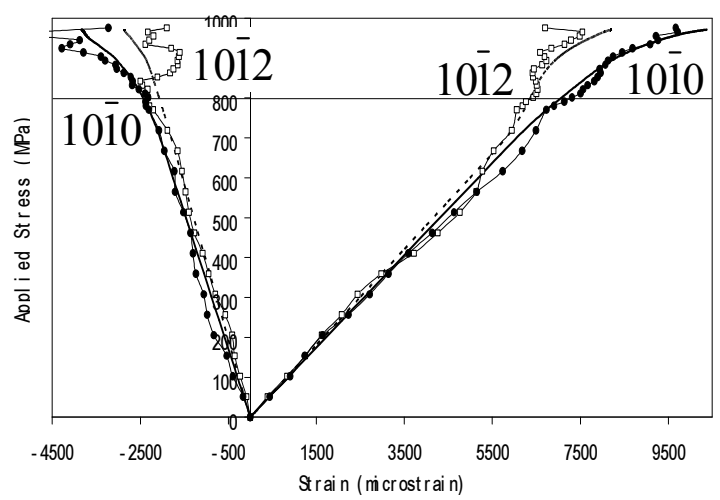

Fig. 4(a). Comparison of FE simulation of Ti64 alloy with the experimental diffraction data, showing macroscopic stress (vertical axis) plotted against the longitudinal and transverse direction $h k l$ microstrains (as indicated).

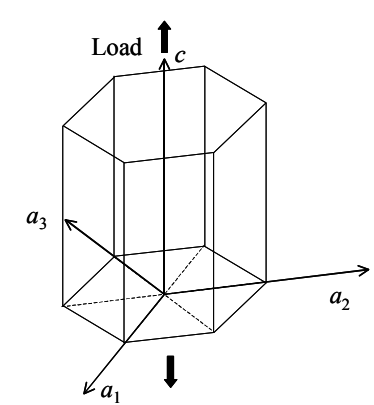

Fig. 4(b). Illustration of the hexagonal lattice cell in the $c$ orientation with respect to the load. Deformation of transverse directions corresponds to $a$-axis strain $\left(\varepsilon_{200}^{T}\right)$.

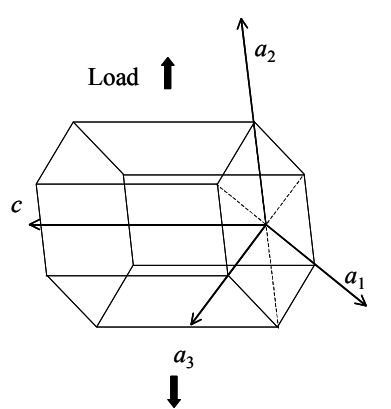

Fig. 4(c). Illustration of the hexagonal lattice cell in the $a$ orientation with respect to the load. Transverse strains correspond to $a$ and $c$ axes ( $\varepsilon_{200}^{T}$ and $\left.\varepsilon_{001}^{T}\right)$. 
The availability of longitudinal and transverse orientation specific elastic lattice strains makes it possible to analyse the residual strains and residual stresses in much more detail, particularly since the fully anisotropic elastic properties of the crystallites can be assumed to be known from the calibration of the polycrystalline FE model.

A series of loading and unloading experiments were also conducted, whereby the initially unstressed sample (unload 0) was loaded in tension to the stress of $930 \mathrm{MPa}$, to cause initial yielding, and then unloaded to zero stress (unload 1). Diffraction patterns were acquired during loading and after unloading. The sample was then re-loaded and unloaded from stress levels of 940 $\mathrm{MPa}$ (2), $950 \mathrm{MPa}$ (3), $960 \mathrm{MPa}$ (4), $970 \mathrm{MPa}$ (5) and $980 \mathrm{MPa}$ (6), with diffraction patterns acquired under load and after unloading. This loading sequence was thought helpful to provide some insight into the development of intergranular residual strains and stresses.

The hexagonal close-packed $\alpha$-phase in the titanium alloy Ti-6Al-4V was assumed to have the following elastic stiffness matrix (in the Voigt notation):

$$
\left[\begin{array}{l}
\sigma_{11} \\
\sigma_{22} \\
\sigma_{33} \\
\sigma_{12} \\
\sigma_{13} \\
\sigma_{23}
\end{array}\right]=C_{L}\left[\begin{array}{c}
\varepsilon_{11} \\
\varepsilon_{22} \\
\varepsilon_{33} \\
2 \varepsilon_{12} \\
2 \varepsilon_{13} \\
2 \varepsilon_{23}
\end{array}\right], \quad C_{L}=\left[\begin{array}{cccccc}
C_{11} & C_{12} & C_{13} & 0 & 0 & 0 \\
C_{12} & C_{11} & C_{13} & 0 & 0 & 0 \\
C_{13} & C_{13} & C_{33} & 0 & 0 & 0 \\
0 & 0 & 0 & C_{44} & 0 & 0 \\
0 & 0 & 0 & 0 & C_{44} & 0 \\
0 & 0 & 0 & 0 & 0 & C_{66}
\end{array}\right],
$$

where $C_{11}=160 \mathrm{GPa}, C_{12}=95 \mathrm{GPa}, C_{13}=45 \mathrm{GPa}, C_{33}=181 \mathrm{GPa}$. The knowledge of this stiffness matrix and its transformation due to grain rotation allows complete determination of the stress state within individual crystallites, provided all the relevant strain components are know either in the global (laboratory) or local (crystal) axes. Further consideration in the present paper will be limited to grains of two particular orientations shown in Fig.s 4(b) and 4(c), respectively.

The orientation shown in Fig. 4(b) is referred to as the $c$-oriented grain, indicating that the crystallographic $c$-axis of the grain coincides with the global load application direction. The orientation shown in Fig. 4(c) is referred to as the $a$-oriented grain, indicating that the crystallographic $a$-axis of the grain (or, in fact, any direction within the basal plane, due to complete isotropy of properties within that plane) coincides with the global load application direction.

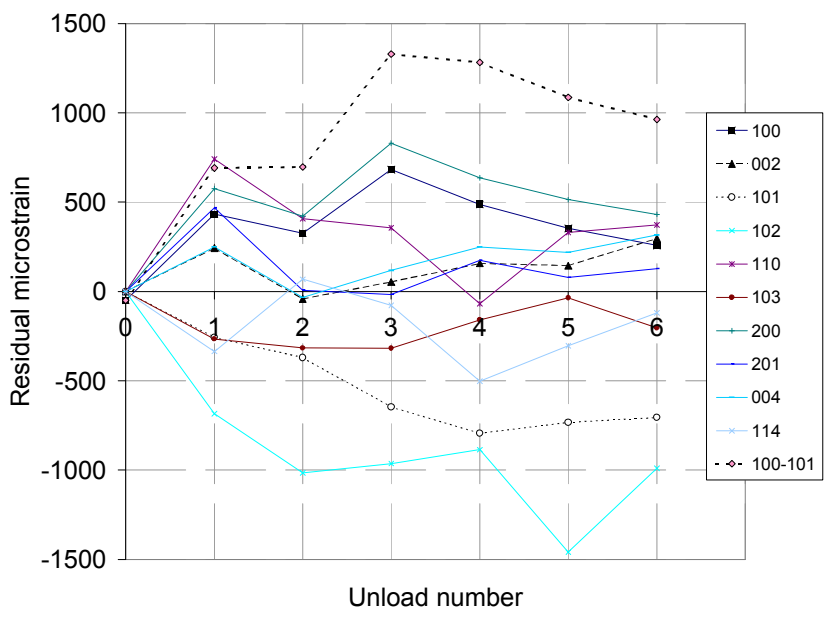

Fig. 5(a). Evolution of residual strains (hk.l), and the difference strain $\varepsilon_{100}-\varepsilon_{001}$, with the number of unload (see text).

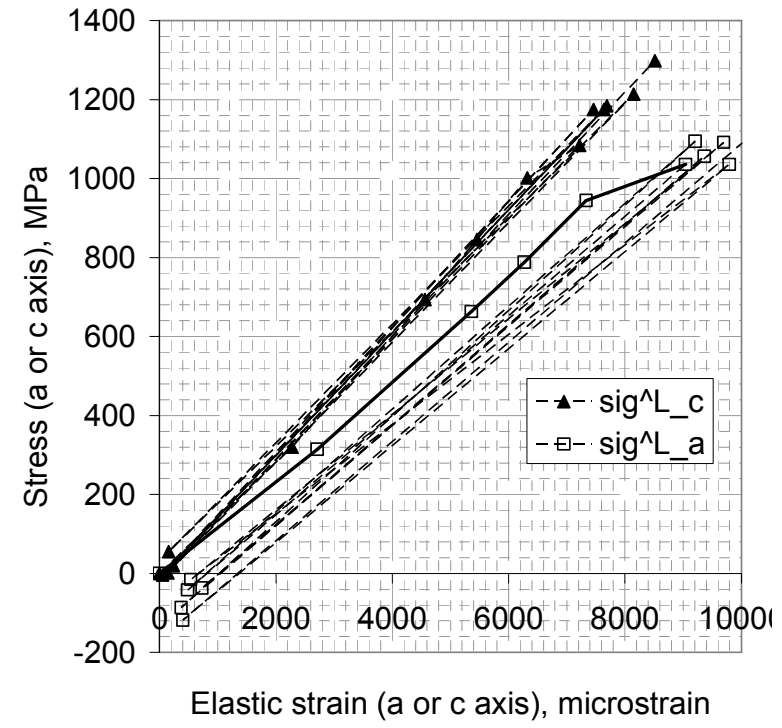

Fig. 5(b). The dependence of orientation-specific microstresses $\sigma_{c}^{L}$ and $\sigma_{a}^{L}$ on their respective elastic strains $\varepsilon_{001}^{L}$ and $\varepsilon_{100}^{L}$, highlighting the difference in the elastic and plastic response of these orientations. 
It is found from the analysis of $h k l$ strains that their evolution between repeated unloads differs greatly depending on the orientation (Fig. 5a). Specifically, (10.0) strains end up in residual tension, while (10.1) strains experience very significant residual compression. In fact, the difference strain $\varepsilon_{100}-\varepsilon_{001}$ acts as an indicator of intergranular strain evolution.

The use of fully anisotropic elastic stiffness matrix, in combination with the complete information about longitudinal and transverse $h k . l$ strains, allows one to calculate the longitudinal orientation-specific microstresses $\sigma_{c}^{L}$ and $\sigma_{a}^{L}$. In Fig. 5(b) microstresses $\sigma_{c}^{L}$ and $\sigma_{a}^{L}$ are plotted against their respective elastic strains $\varepsilon_{001}^{L}$ and $\varepsilon_{100}^{L}$, highlighting the difference in elastic (slope) and plastic (non-linearity) responses of grain orientations considered.

\section{Discussion and conclusions}

Several aspects of the general approach to the analysis of polycrystal deformation have been presented in this paper. Firstly, the polycrystal elasto-plasticity finite element modelling formulation was introduced, followed by the description of experiment setup that allows the measurement of orientation-specific mesoscopic average strains. Finally, a procedure for the interpretation of these results in terms of microstrains and microstresses has been outlined.

The results obtained from both the modelling framework and the interpretation of experimental data highlight the importance of elastic and plastic inhomogeneity at the crystallite level for the development of intergranular residual strains and stresses. The existence of grain orientations leading to extremes of behaviour can be readily identified, either from the analysis of numerical simulations, or from careful interpretation of experimental measurements.

The differences in the deformation response of these extreme groups of grains are likely to play an important role in determining the resistance of the entire polycrystal assembly to crack initiation under the action of cyclic external loading. These phenomena a closely linked to the energy dissipation processes at the grain level, and therefore to the process of damage accumulation. Thus, detailed studies of integranular strains and stresses may, in the authors opinion, lead to the formulation of improved, more details criteria for crack initiation under fatigue conditions, providing the basis for more reliable and efficient design of engineering components and structures.

\section{Acknowledgements}

The authors wish to thank Dr Ed Oliver (ENGIN-X, ISIS facility, Rutherford Appleton Laboratory, near Oxford) and Drs Matthew Peel, Thomas Buslaps and Veijo Honkimaki (ID15, European Synchrotron Radiation Facility, Grenoble, France) for their invaluable help in setting up in situ loading experiments and conducting diffraction measurements with neutron and high energy X-ray beams.

\section{References}

[1] A. Manonukul and F. P. E. Dunne: Proc. R. Soc. Lond. A460 (2004) p. 1881-1903.

[2] R. Hill and J. R. Rice: J. Mech. Phys. Solids., 20 (1972) p. 401.

[3] R. J. Asaro: Advances in Applied Mechanics, 23 (1983) p. 1-115.

[4] A. M. Korsunsky, D. Dini, F. P. E. Dunne, and M. J. Walsh: Intl J Fatigue, 29 (2007) p.19901995, http://dx.doi.org/10.1016/j.ijfatigue.2007.01.007

[5] J. Liu, K. Kim, M. Golshan, D. Laundy, A.M. Korsunsky: J.Appl. Cryst. 38 (2005) p.661-667.

[6] M.R. Daymond, M.A.M. Bourke, R.B. Von Dreele, B. Clausen, T. Lorentzen: J. Appl. Phys., 82 (1007) p.1554-1562.

[7] A.M. Korsunsky, M.R. Daymond, K.E. James: Materials Science Engng A334 (2002) p.4148. 